\title{
Effect of Cr Content on the Properties of Magnetic Field Processed Cr-Doped ZnO-Diluted Magnetic Semiconductors
}

\author{
Shiwei Wang, Weiqiang Bo, Min Zhong, Cong Liu, Ying Li, Mingyuan Zhu, Yemin Hu, \\ and Hongmin Jin
}

Laboratory for Microstructures, School of Materials Science and Engineering, Shanghai University, 149 Yanchang Road, Shanghai 200072, China

Correspondence should be addressed to Shiwei Wang, wenruru@sina.com

Received 9 May 2012; Accepted 5 June 2012

Academic Editor: Renzhi Ma

Copyright (๑) 2012 Shiwei Wang et al. This is an open access article distributed under the Creative Commons Attribution License, which permits unrestricted use, distribution, and reproduction in any medium, provided the original work is properly cited.

Cr-doped ZnO-diluted magnetic semiconductor (DMS) nanocrystals with various Cr contents were synthesized by hydrothermal method under high magnetic field. The result indicated that both the amount of Cr contents and high magnetic field significantly influenced crystal structure, morphology, and magnetic property of $\mathrm{Cr}$-doped $\mathrm{ZnO}$ DMSs. When the Cr contents increased from 1 at $\%$ to 5 at $\%$, the morphology of grains sequentially changed from flower-like to rod-like and then to the flake-like form. All the samples remained hexagonal wurtzite structure after $\mathrm{Cr}$ ions were doped into the $\mathrm{ZnO}$ crystal lattice. The $\mathrm{Cr}$ doping led to the increasing amount of defects and even enhanced the magnetic property of the matrix materials. All the Cr-doped ZnO DMSs obtained under high magnetic field exhibited obvious ferromagnetic behavior at room temperature. The results have also shown the successful substitution of the $\mathrm{Cr}^{3+}$ ions for the $\mathrm{Zn}^{2+}$ ions in the crystal lattice.

\section{Introduction}

Recently the interest in diluted magnetic semiconductors (DMSs) has significantly increased because of their unique magnetic property and potential applications in many fields [1-6]. Ferromagnetic DMSs with Curie temperatures (Tc) above room temperature are usually obtained by doping magnetic ions into the host semiconductors. Zinc oxide is one of representative semiconductor $[7,8]$, due to its wide bandgap of $3.37 \mathrm{eV}$ and large exciton energy $(60 \mathrm{meV})$. As a transition metal ion, $\mathrm{Cr}^{3+}$ is often used as a dopant for $\mathrm{ZnO}$ because of the close ionic radius with $\mathrm{Zn}^{2+}$ and the ferromagnetic stability of Cr-doped ZnO DMSs $[9,10]$.

Currently, many methods have been reported to fabricate $\mathrm{ZnO}$ DMSs, such as vapor-phase growth [11], thermal decomposition [12], seed-mediated [13], and reverse micelle [14]. Recently, our group has successfully developed a novel hydrothermal method under high magnetic field to prepare a series of transition metal ions $\left(\mathrm{Cr}^{3+}, \mathrm{Mn}^{2+}, \mathrm{Co}^{2+}\right)$ doped $\mathrm{ZnO}$ DMSs. According to our previous work, it revealed that high magnetic field played an important role in introducing ions into $\mathrm{ZnO}$ crystal lattice and improving the magnetic properties [15-17]. In the present work, the effect of $\mathrm{Cr}$ content on the structure, morphology, and magnetic property of Cr-doped ZnO DMSs prepared by HPMF method was investigated in detail.

\section{Experimental}

A hydrothermal method with pulsed magnetic field of 4 Tesla ( $\mathrm{T}$ ) was used to synthesize the Cr-doped $\mathrm{ZnO}$ DMS materials with different $\mathrm{Cr}$ nominal contents ( 1 at $\%, 2$ at $\%$, 3 at $\%, 5$ at $\%$ ). The typical synthesis process was as follows. A $12 \mathrm{~mL} 1.33 \mathrm{M}$ solution of potassium hydroxide was slowly dropped into a solution of $4 \mathrm{~mL}$ of $1.00 \mathrm{M}$ zinc acetate. The mixture was stirred at $273 \mathrm{~K}$ for 0.5 hours. Then, a desired amount of $0.02 \mathrm{M}$ chromium nitrate solution, which was calculated according to the nominal Cr content, was slowly dropped into the former mixture at $273 \mathrm{~K}$ in the ice bath, and followed by stirring for $1 \mathrm{~h}$. The entire mixtures with 


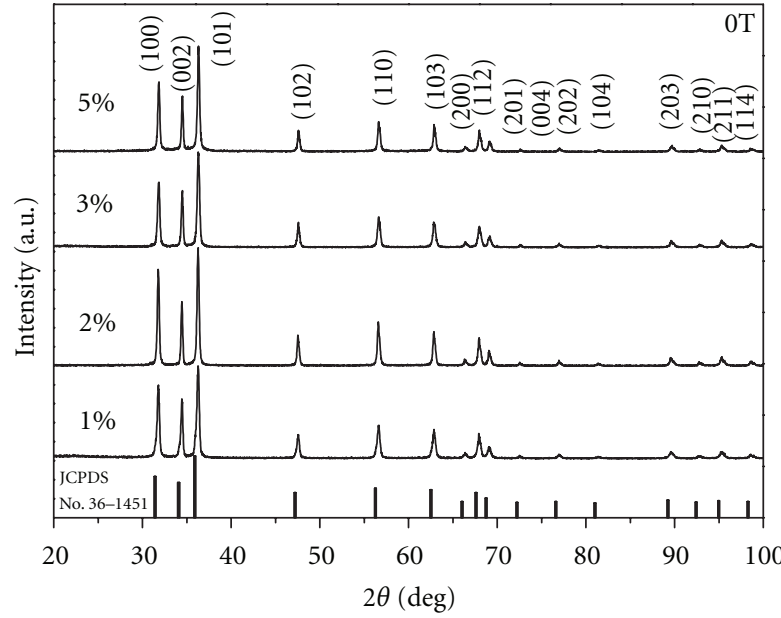

(a)

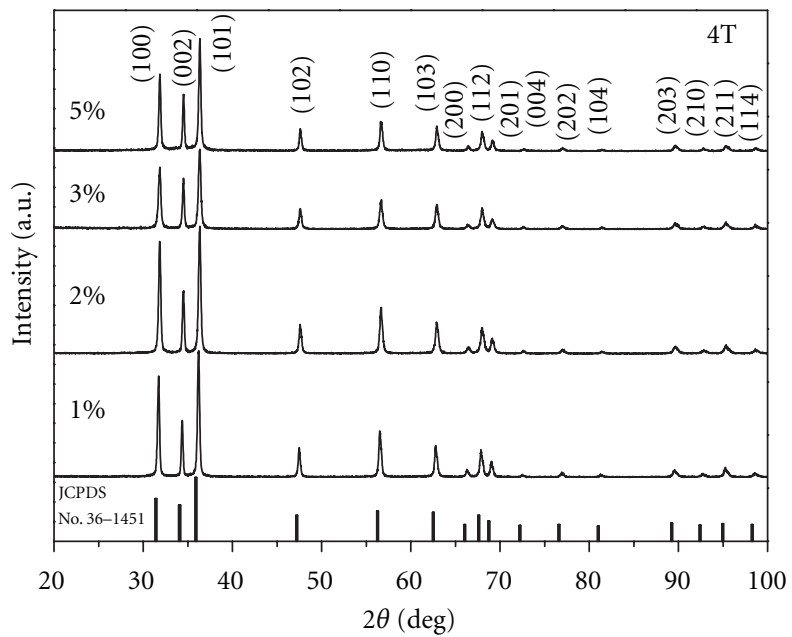

(c)

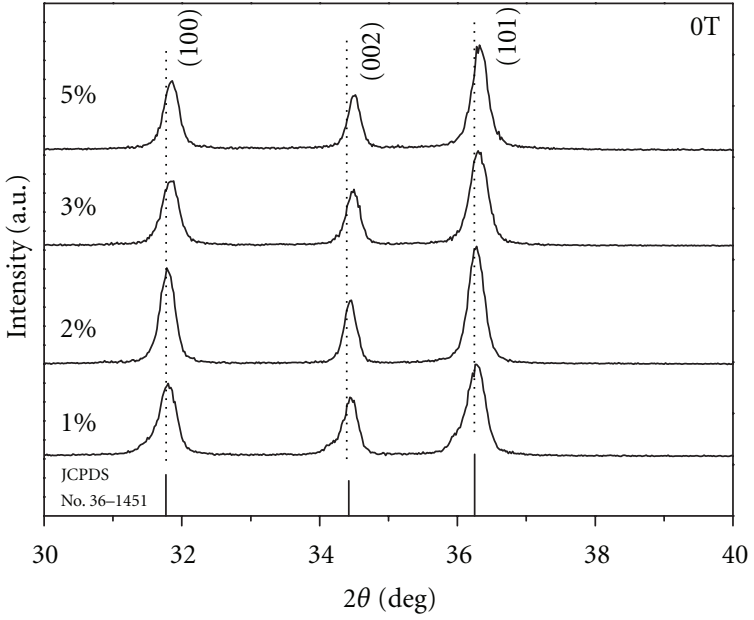

(b)

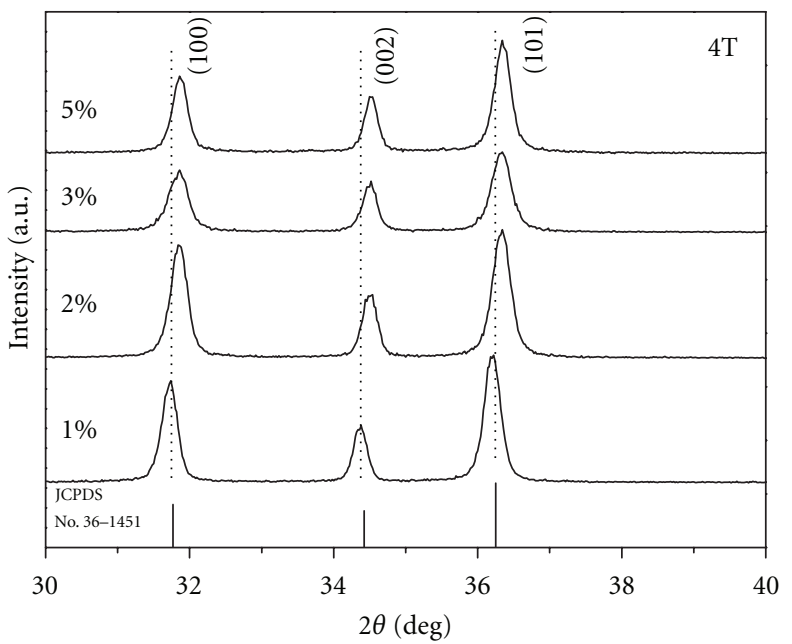

(d)

FIGURE 1: XRD patterns of 1\%, 2\%, 3\%, and 5\% Cr-doped ZnO synthesized under $0 \mathrm{~T}((\mathrm{a}),(\mathrm{b}))$ and $4 \mathrm{~T}((\mathrm{c}),(\mathrm{d}))$ magnetic fields.

a certain concentration of chromium nitrate in zinc acetate solution were then transferred into autoclave $(25 \mathrm{~mL})$ and maintained at $453 \mathrm{~K}$ for $4 \mathrm{~h}$ with $4 \mathrm{~T}$ pulsed magnetic field (the obtained sample named as $4 \mathrm{~T}-\mathrm{N} \%$ ) or without pulsed magnetic field (named as $0 \mathrm{~T}-\mathrm{N} \%$ ), respectively, in which $N$ presents the $\mathrm{Cr}$ nominal content. Finally, the precipitated products were washed with distilled water three times and dried at $373 \mathrm{~K}$ for $10 \mathrm{~h}$.

The crystal structures of the samples were determined by X-ray diffraction (XRD) equipped with $\mathrm{CuK}_{\alpha}$ radiation $(\lambda=1.5406 \AA)$. Field emission scanning electron microscope (FE-SEM, JEOL JSM-6700) was used to observe the morphologies. The Raman scattering spectra of the samples were recorded by a Renishaw InVia Confocal micro-Raman system using the $785 \mathrm{~nm}$ line as excitation source. Magnetic property of the samples was measured by vibrating sample magnetometer (VSM, Lakeshore 7407). The valence state of the $\mathrm{Cr}$ element was analyzed by X-ray photoelectron spectroscopy (XPS) (Thermo ESCALAB 250).

\section{Results and Discussion}

Figure 1 shows the XRD patterns of the Cr-doped ZnO DMSs with various $\mathrm{Cr}$ contents synthesized with and without $4 \mathrm{~T}$ magnetic fields. All the diffraction peaks (Figures 1(a) and $1(c))$ are sharp and match well with those of hexagonal wurtzite structure of $\mathrm{ZnO}$ (space group: P63mc (186), JCPDS no. 36-145). No Cr, Cr oxides, or any other impurityphase peaks are observed which reveals that $\mathrm{Cr}$-doped $\mathrm{ZnO}$ DMSs maintain a highly pure crystalline wurtzite structure. Comparing the diffraction peaks (100), (002), and (101) of Cr-doped ZnO DMSs (Figures 1(b) and 1(d)), the peaks shift to larger angles with the increase of $\mathrm{Cr}$ contents and the highest shift is for sample with $5 \% \mathrm{Cr}$ nominal content. The reason is due to the smaller ion radius of $\mathrm{Cr}^{3+}(0.63 \AA)$ comparing to that of $\mathrm{Zn}^{2+}(0.74 \AA)$. The lattice constants of $\mathrm{ZnO}$ decrease slightly after $\mathrm{Cr}$ ions doped into the crystal lattice which cause the main peaks moving to the higher angles. The higher the content of $\mathrm{Cr}$ ions in the $\mathrm{ZnO}$ lattice, the farther the peaks move. Furthermore, the diffraction 


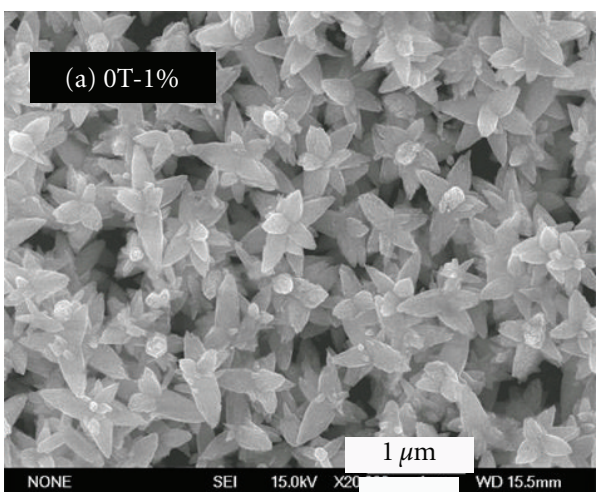

(a)

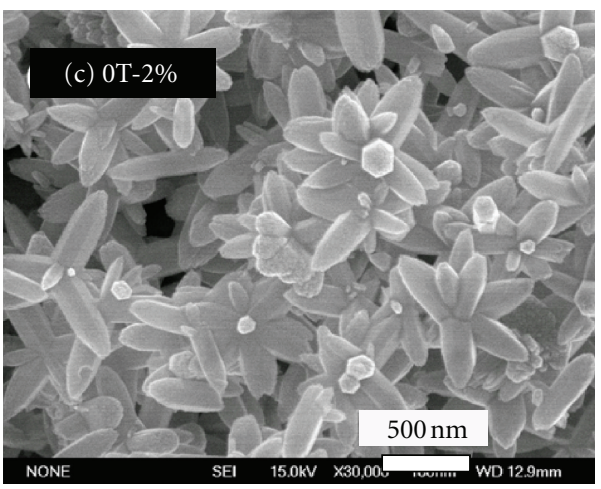

(c)

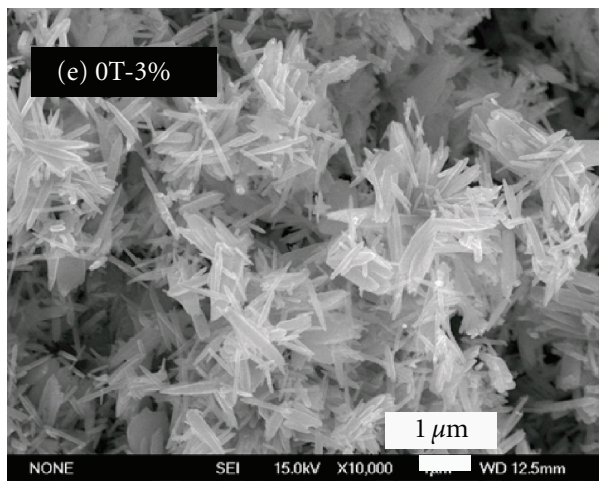

(e)

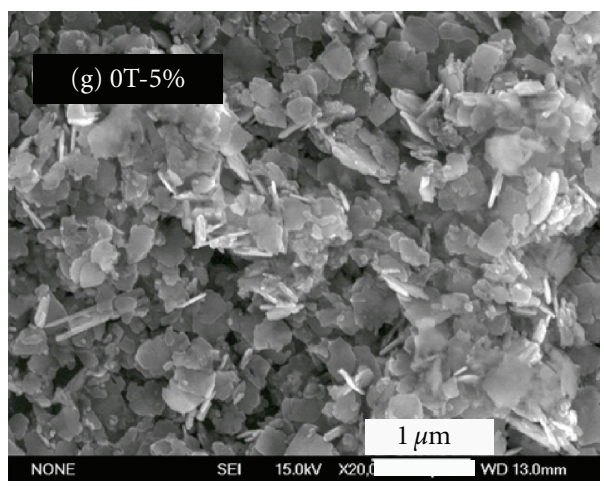

(g)

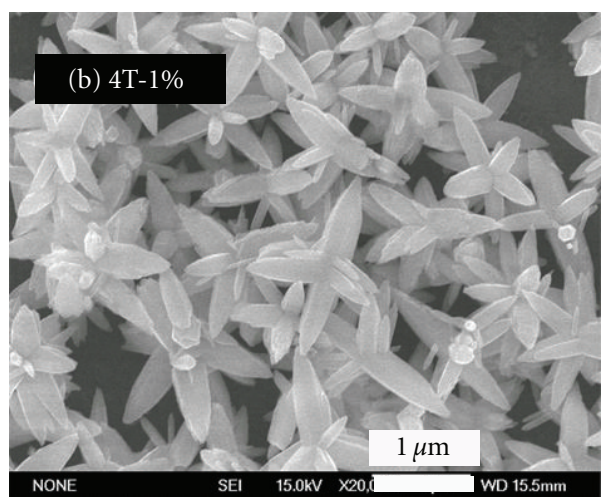

(b)

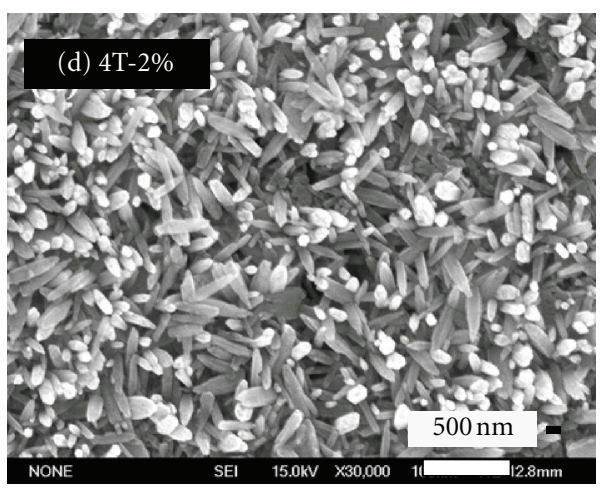

(d)

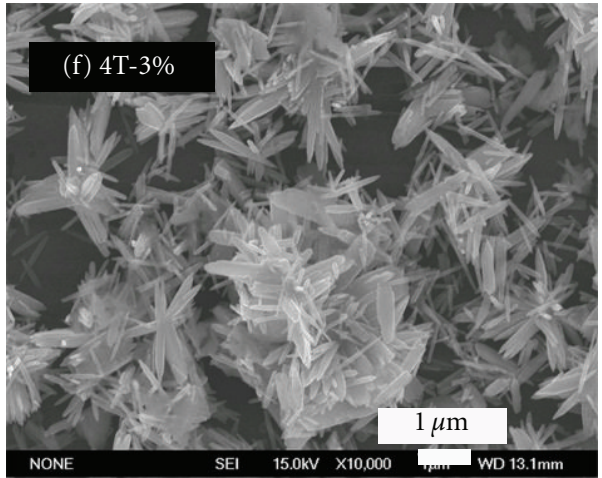

(f)

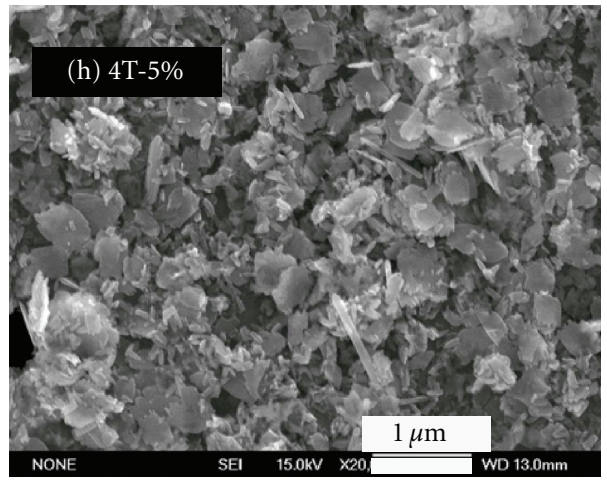

(h)

Figure 2: SEM images of Cr-doped $\mathrm{ZnO}$ with different Cr nominal content synthesized under $0 \mathrm{~T}$ ((a), (c), (e), (g)) and 4 T magnetic fields ((b), (d), (f), (h)). 


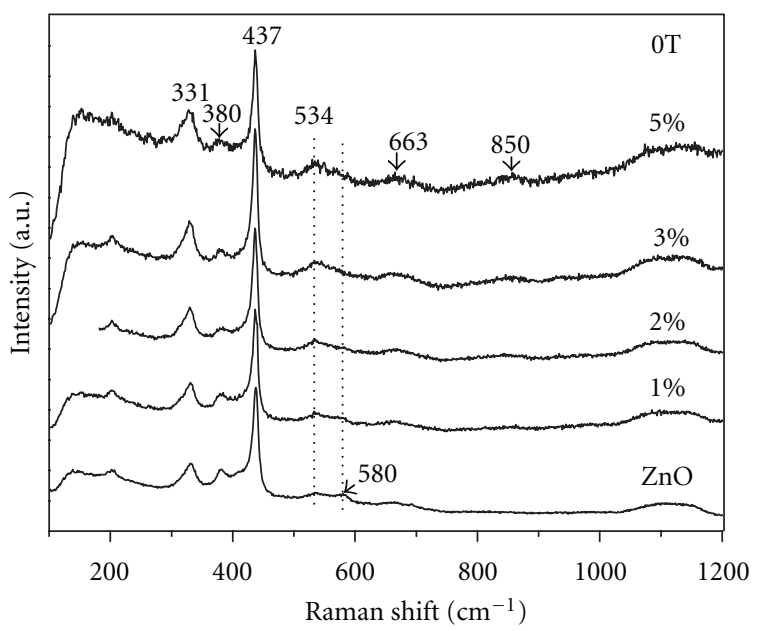

(a)

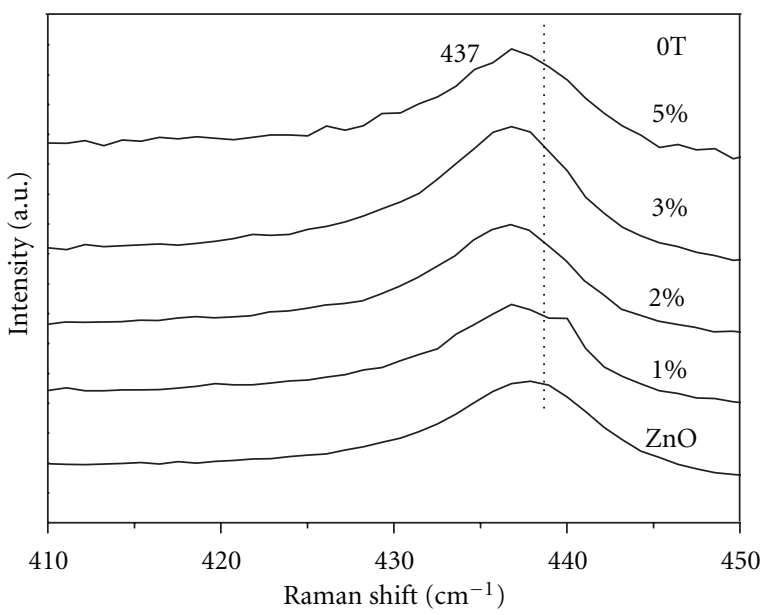

(c)

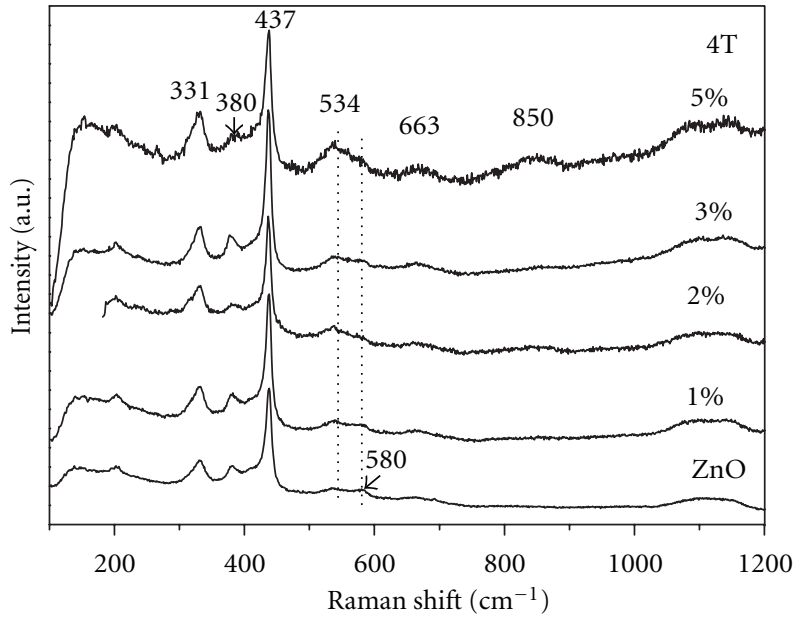

(b)

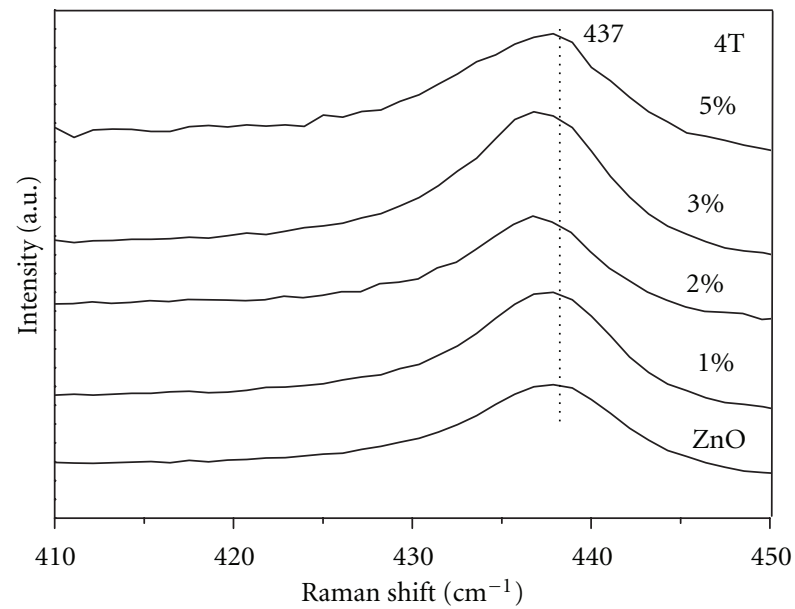

(d)

Figure 3: Raman spectra of Cr-doped ZnO DMSs with different Cr nominal contents obtained under $0 \mathrm{~T}$ ((a), (c)) and $4 \mathrm{~T}$ ((b), (d)) magnetic fields, respectively.

peaks (100), (002), and (101) of samples synthesized under $4 \mathrm{~T}$ magnetic field move to larger angles comparing with those of the same Cr content samples synthesized without magnetic field, which indicates that, under high magnetic field, larger amount of $\mathrm{Cr}$ ions were doped into the $\mathrm{ZnO}$ lattice.

Figure 2 shows the SEM images of $\mathrm{Cr}$-doped $\mathrm{ZnO}$ with various $\mathrm{Cr}$ nominal contents synthesized under $0 \mathrm{~T}$ and $4 \mathrm{~T}$ magnetic fields. It is observed that with the Cr contents' increase from $1 \%$ to $5 \%$, the morphologies of particles change from flower shape to rod shape and eventually to flake shape. For samples with $\mathrm{Cr}$ nominal content of $1 \%$ (Figures $2(\mathrm{a})$ and $2(\mathrm{~b})$ ), the grains are both flower shape, but the sizes are different, as sizes of the $4 \mathrm{~T}-1 \%$ are much smaller than that of $0 \mathrm{~T}-1 \%$. However, for the $2 \% \mathrm{Cr}$ content samples (Figures $2(\mathrm{c})$ and $2(\mathrm{~d})$ ), both crystalline shapes and sizes are different, the $4 \mathrm{~T}-2 \%$ sample appears as fine dense rod shape with rod diameter of 50-100 nm, whereas the 0T-2\% sample appears as flower shape with rod diameter of $100-250 \mathrm{~nm}$. Both of the samples with $\mathrm{Cr}$ nominal content of $3 \%$ (Figures 2(e) and 2(f)) appear with irregular rod shape with a little difference in particles size. When the Cr nominal content reaches 5\% (Figures 2(g) and 2(h)), both samples appear with irregular flake shapes, while the $4 \mathrm{~T}-5 \%$ sample shows more small grains than the $0 \mathrm{~T}-5 \%$ sample. Clearly, the morphologies of zinc oxides grains are influenced dramatically as the $\mathrm{Cr}$ content increased. Concurrently high magnetic field also plays a significant role in the morphologies of Cr-doped ZnO DMSs. Tanaka et al. [18] reported that $c$-axis-oriented $\mathrm{ZnO}$ could be fabricated in a rotating sample container under static magnetic field during the hydrothermal doping process. The high pulsed magnetic field essentially provided an external energy and promoted the nucleation and growth of the grains, which finally caused the differentiation of crystal morphology.

All the EDS data (Table 1) verify that the actual incorporated $\mathrm{Cr}$ contents are lower than the corresponding $\mathrm{Cr}$ nominal contents in the doping process, and increase 


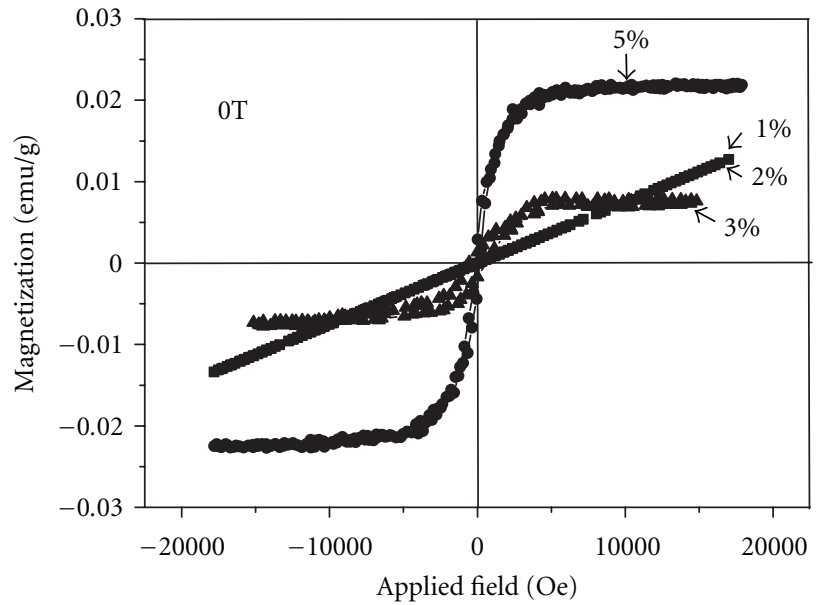

(a)

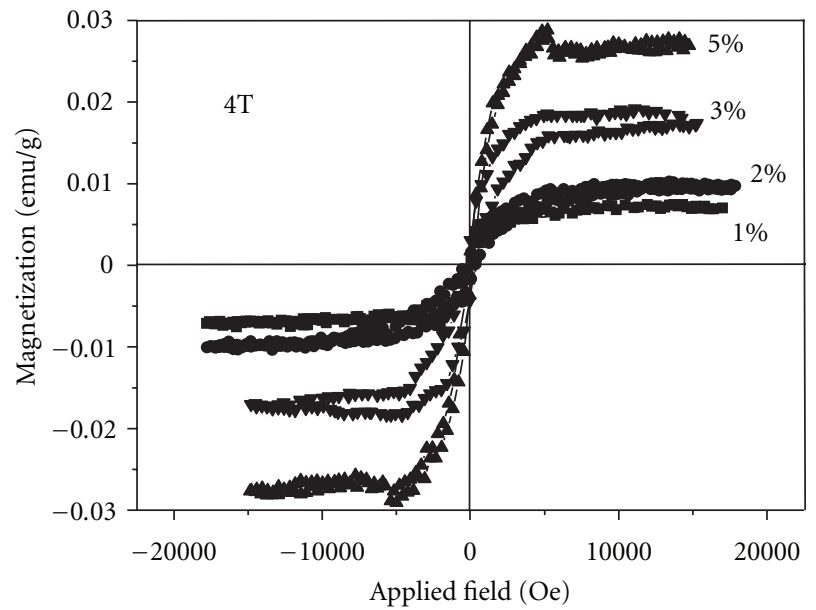

(b)

FIgure 4: Hysteresis loops of Cr-doped ZnO DMSs with different Cr nominal contents synthesized under $0 \mathrm{~T}$ (a) and $4 \mathrm{~T}$ (b) magnetic fields, respectively.

nominal $\mathrm{Cr}$ contents does increase the actual incorporation of $\mathrm{Cr}$. Furthermore, the incorporation of $\mathrm{Cr}$ into the $\mathrm{ZnO}$ crystal lattice is greater with applying magnetic field than without, which agrees with the results of XRD.

Raman scattering is a versatile technique to characterize the incorporation of $\mathrm{Cr}$ ions and the defects in the host lattice. The peak at about $437 \mathrm{~cm}^{-1}$ assigned to the $E_{2}$ (high) mode of $\mathrm{ZnO}$, which is the characteristic mode of the wurtzite structure and sensitive to internal stress [19]. The peak at $580 \mathrm{~cm}^{-1}$ assigned to $A_{1}$ (LO) mode, which is sensitive to changes in the free carrier concentration [20]. From Figures 3(a) and 3(b), there is no obvious observed shift at $437 \mathrm{~cm}^{-1}$ for all samples. But compared to the pure $\mathrm{ZnO}$, there is a blue shift with the increase of the Cr doping as shown in the magnified Raman shift region as shown in Figures 3(c) and 3(d). These indicate that with the increase of Cr doping, a little structure disorder was occurred and the little blue shift of the peak also illustrates that the lower amount of $\mathrm{Cr}$ doping would not change the crystal structure significantly although defects was introduced by $\mathrm{Cr}$ doping. Additionally, when Cr ions were doped, the Raman peaks were slightly changed for the $1 \%, 2 \%, 3 \%$ samples, and comparing to the characteristic peaks of pure $\mathrm{ZnO}$ the peak at $580 \mathrm{~cm}^{-1}$ gradually disappeared. The gradually disappearance of the $580 \mathrm{~cm}^{-1}$ peak is created by the increase of carrier concentration. When $\mathrm{Cr}$ ions increased to 5\%, the peak at $534 \mathrm{~cm}^{-1}$ increased disproportionally and a new peak appeared at $850 \mathrm{~cm}^{-1}$. This new phenomenon is rather unique for the $4 \mathrm{~T}-5 \%$ sample only. All these spectroscopic changes probably indicated that the increase of $\mathrm{Cr}$ ions incorporation also increases the defect and structures of the $\mathrm{ZnO}$ nanocrystals.

Figure 4 shows the magnetic hysteresis $(\mathrm{M}-\mathrm{H})$ loops of the $\mathrm{Cr}$-doped $\mathrm{ZnO}$ with various $\mathrm{Cr}$ nominal contents synthesized under $0 \mathrm{~T}$ and $4 \mathrm{~T}$ magnetic fields, respectively. It can be seen from Figure $4(\mathrm{a})$ that $0 \mathrm{~T}-1 \%$ and $0 \mathrm{~T}-2 \%$ samples show paramagnetic behavior, while $0 \mathrm{~T}-3 \%$ and $0 \mathrm{~T}-5 \%$
TABLE 1: EDS parameters and saturation magnetization of Crdoped ZnO DMSs synthesized with or without high magnetic fields.

\begin{tabular}{ccccc}
\hline Cr nominal & \multicolumn{2}{c}{ Actual Cr content (at\%) } & \multicolumn{2}{c}{ Ms (emu/g) } \\
content (at\%) & $0 \mathrm{~T}$ & $4 \mathrm{~T}$ & $0 \mathrm{~T}$ & $4 \mathrm{~T}$ \\
\hline 1 & 0.22 & 0.35 & - & 0.0075 \\
2 & 1.1 & 1.4 & - & 0.01 \\
3 & 2.3 & 2.4 & 0.01 & 0.02 \\
5 & 3.1 & 3.7 & 0.02 & 0.03 \\
\hline
\end{tabular}

samples show ferromagnetic behavior at room temperature. It had been discussed in our previous work [15] that the weak hybridization effect between $\mathrm{Cr}$ ions and defects is the reason which results in paramagnetic behavior of sample $0 \mathrm{~T}-2 \%$ at 298 K. From Figure 4(b), all the samples obtained under $4 \mathrm{~T}$ magnetic fields manifest room temperature ferromagnetic behavior. The saturation magnetizations (Ms, Table 1) of $1 \%$, $2 \%, 3 \%$, and $5 \%$ sample under pulsed high magnetic field was enhanced compared with $0 \mathrm{~T}$ samples, respectively. It is believed that the occurrence of strong electronic coupling between bound polarons and $\mathrm{Cr}$ ions forms bound magnetic polarons (BMPs) [3], which is proposed to play an important role in the ferromagnetic origin of Cr-doped ZnO DMSs [21, 22]. Kittilstved et al. [23] suggested defect-bound carriers such that point defects hybridization with magnetic dopants would induce ferromagnetism in transition metal ions doped $\mathrm{ZnO}$. In this study, we have found that, by applying high magnetic field, it can increase the incorporation of $\mathrm{Cr}$ ions into the $\mathrm{ZnO}$ crystal lattice, and this is shown by the XRD, SEM, and Raman analysis. Since more defects are introduced into the crystal lattice under high magnetic field, the hybridization between defects and $\mathrm{Cr}$ dopants should be more efficient, which is probably the cause of room temperature ferromagnetism and the enhancement of saturation magnetization of the Cr-doped ZnO DMSs. 


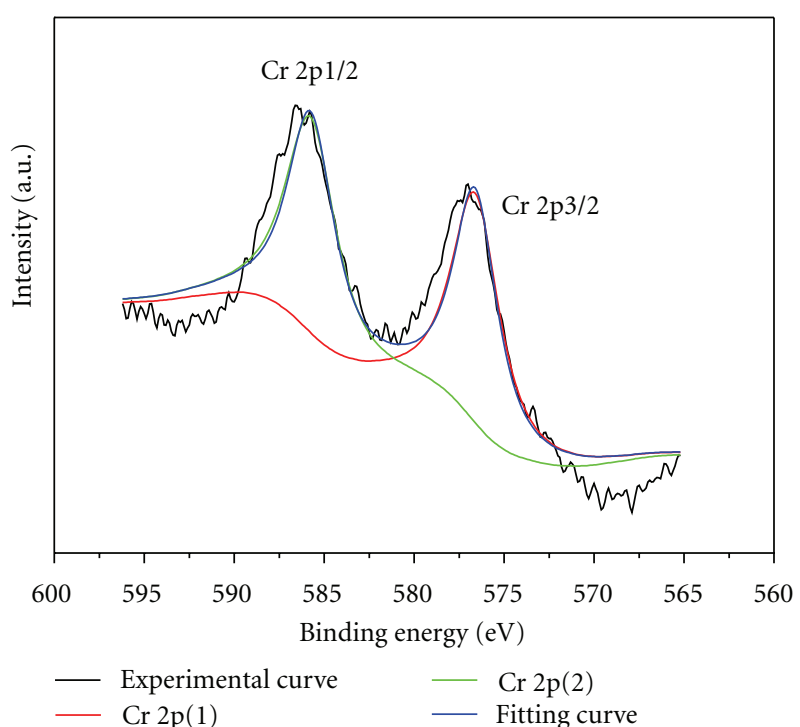

(a)

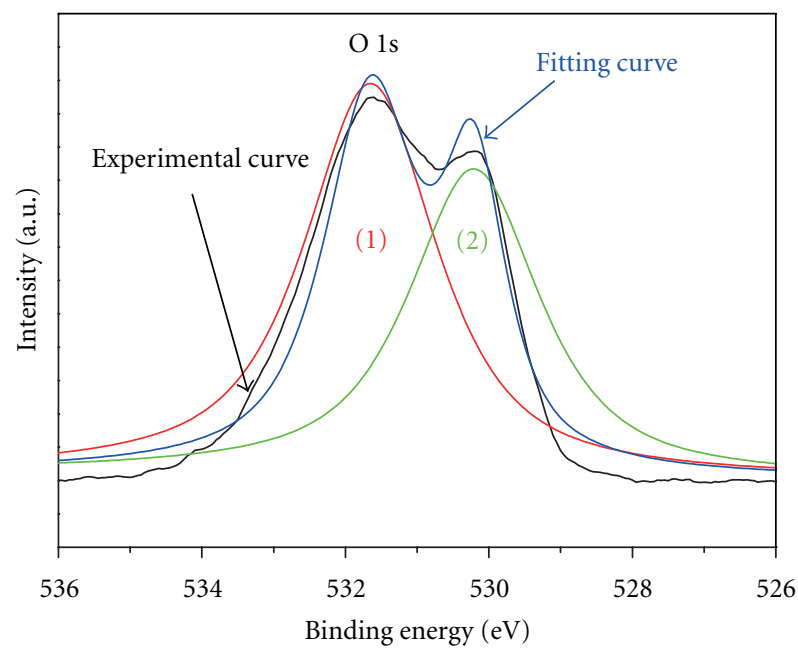

(b)

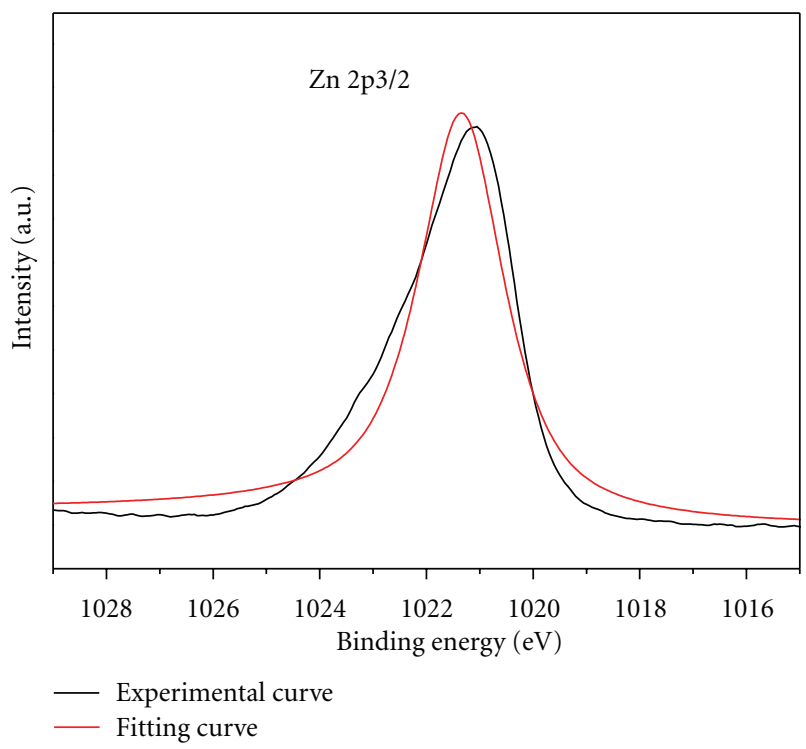

(c)

FIGURE 5: XPS spectrum of Cr-doped $\mathrm{ZnO}$ nanoparticles.

In order to investigate the chemical state of the host and dopant elements, the XPS studies were undertaken. XPS analysis shows the presence of $\mathrm{Zn}, \mathrm{Cr}$, and $\mathrm{O}$ elements in $\mathrm{Cr}$-doped $\mathrm{ZnO}$. The overlapped bands were resolved into separated peaks by using XPS PEAK41 software. The $\mathrm{Cr}$ 2p3/2, Cr 2p1/2, O 1s, and Zn 2p3/2 XPS spectral regions of the $\mathrm{Cr}$-doped $\mathrm{ZnO}$ nanoparticle are shown in Figure 5 for $1 \% \mathrm{Cr}-4 \mathrm{~T}$ sample. Based on the Gauss fitting, the $\mathrm{Cr}$ $2 \mathrm{p} 3 / 2$ peak position is at $576.6 \mathrm{eV}$ in the $\mathrm{Cr}$-doped $\mathrm{ZnO}$ nanoparticle. It is clearly different from $574.2 \mathrm{eV}$ of $\mathrm{Cr}$ metal and $576.0 \mathrm{eV}$ of $\mathrm{Cr}^{2+}$, but it is quite close to the peak position of $\mathrm{Cr} 2 \mathrm{p} 3 / 2(576.7 \mathrm{eV})$ in $\mathrm{Cr}_{2} \mathrm{O}_{3}$ [24]. It suggests that the $\mathrm{Cr}$ dopants in the $\mathrm{ZnO}$ lattice are actually the $\mathrm{Cr}^{3+}$ ions.

\section{Conclusions}

The 1\%, 2\%, 3\%, and 5\% Cr-doped ZnO DMSs nanocrystals were synthesized by hydrothermal method under high magnetic field. Both the $\mathrm{Cr}$ content and high magnetic field significantly impacted the microstructure and magnetic property of Cr-doped $\mathrm{ZnO}$ DMSs. With the increase of $\mathrm{Cr}$ contents from $1 \%$ to $5 \%$, the morphologies of particles changed from flower shape to rod shape and eventually to flake shape. After the incorporation of $\mathrm{Cr}$, the $\mathrm{ZnO}$ DMSs nanocrystals still retained wurtzite crystal structure. The $\mathrm{Cr}$ doping caused the increased amount of defects and the enhancement of magnetic property of the materials. All the Cr-doped $\mathrm{ZnO}$ DMSs obtained under high magnetic field exhibited ferromagnetic behavior at room temperature. The 
$\mathrm{Cr}$ ions that incorporated in the $\mathrm{ZnO}$ lattice were shown to be the $\mathrm{Cr}^{3+}$ cations.

\section{Acknowledgments}

Shanghai Science and Technology Commission (11nm 0501600, 09dz1203602), Shanghai Leading Academic Discipline Project (S30107), and the Instrumental Analysis and Research Center of Shanghai University are acknowledged for their technical supports.

\section{References}

[1] H. Ohno, "Making nonmagnetic semiconductors ferromagnetic," Science, vol. 281, no. 5379, pp. 951-956, 1998.

[2] K. R. Kittilstved, W. K. Liu, and D. R. Gamelin, "Electronic structure origins of polarity-dependent high-Tc ferromagnetism in oxide-diluted magnetic semiconductors," Nature Materials, vol. 5, no. 4, pp. 291-297, 2006.

[3] J. M. D. Coey, M. Venkatesan, and C. B. Fitzgerald, "Donor impurity band exchange in dilute ferromagnetic oxides," Nature Materials, vol. 4, no. 2, pp. 173-179, 2005.

[4] A. Samariya, R. K. Singhal, S. Kumar et al., "Defect-induced reversible ferromagnetism in Fe-doped $\mathrm{ZnO}$ semiconductor: an electronic structure and magnetization study," Materials Chemistry and Physics, vol. 123, no. 2-3, pp. 678-684, 2010.

[5] R. K. Singhal, A. Samariya, Y. T. Xing et al., "Electronic and magnetic properties of $\mathrm{Co}$-doped $\mathrm{ZnO}$ diluted magnetic semiconductor," Journal of Alloys and Compounds, vol. 496, no. 1-2, pp. 324-330, 2010.

[6] R. K. Singhal, A. Samariya, S. Kumar et al., "Switch "on" and "off" ferromagnetic ordering through the induction and removal of oxygen vacancies and carriers in doped $\mathrm{ZnO}$ : a magnetization and electronic structure study," Physica Status Solidi (A) Applications and Materials, vol. 207, no. 10, pp. 2373-2386, 2010.

[7] J. H. Lim, C. K. Kong, K. K. Kim, I. K. Park, D. K. Hwang, and S. J. Park, "UV electroluminescence emission from $\mathrm{ZnO}$ lightemitting diodes grown by high-temperature radiofrequency sputtering," Advanced Materials, vol. 18, no. 20, pp. 27202724, 2006.

[8] J. K. Furdyna, "Diluted magnetic semiconductors," Journal of Applied Physics, vol. 64, no. 4, pp. R29-R64, 1988.

[9] T. Dietl, T. Dietl, H. Ohno, F. Matsukura, J. Cibert, and D. Ferrand, "Zener model description of ferromagnetism in zincblende magnetic semiconductors," Science, vol. 287, no. 5455, pp. 1019-1022, 2000.

[10] K. Sato and H. Katayama-Yoshida, "Material design for transparent ferromagnets with $\mathrm{ZnO}$-based magnetic semiconductors," Japanese Journal of Applied Physics, Part 2, vol. 39, no. 6, pp. L555-L558, 2000.

[11] D. C. Kundaliya, S. B. Ogale, S. E. Lofland et al., "On the origin of high-temperature ferromagnetism in the low-temperatureprocessed Mn-Zn-O system," Nature Materials, vol. 3, no. 10, pp. 709-714, 2004.

[12] A. Quesada, M. A. García, P. Crespo, and A. Hernando, "Materials for spintronic: room temperature ferromagnetism in Zn-Mn-O interfaces," Journal of Magnetism and Magnetic Materials, vol. 304, no. 1, pp. 75-78, 2006.

[13] H. B. Wang, H. Wang, C. Zhang et al., "Effect of annealing on the magnetic properties of solution synthesized $\mathrm{Zn1-xMnxO}$ nanorods," Materials Chemistry and Physics, vol. 113, no. 2-3, pp. 884-888, 2009.

[14] D. Milivojević, J. Blanuša, V. Spasojević, V. Kusigerski, and B. Babić-Stojić, "Room temperature ferromagnetism in $\mathrm{Zn}-\mathrm{Mn}$ O," Solid State Communications, vol. 141, no. 12, pp. 641-644, 2007.

[15] Y. Li, Y. Li, M. Zhu et al., "Structure and magnetic properties of $\mathrm{Cr}$-doped $\mathrm{ZnO}$ nanoparticles prepared under high magnetic field," Solid State Communications, vol. 150, no. 15-16, pp. 751-754, 2010.

[16] T. Yang, Y. Li, M. Y. Zhu et al., "Room-temperature ferromagnetic Mn-doped $\mathrm{ZnO}$ nanocrystal synthesized by hydrothermal method under high magnetic field," Materials Science and Engineering B, vol. 170, no. 1-3, pp. 129-132, 2010.

[17] J. Huang, M. Zhu, Y. Li et al., "Fabricating Co doped $\mathrm{ZnO}$ nanocrystallines by hydrothermal method with high pulsed magnetic field," Journal of Nanoscience and Nanotechnology, vol. 10, no. 11, pp. 7303-7306, 2010.

[18] S. Tanaka, A. Makiya, Z. Kato, and K. Uematsu, "c-axis oriented $\mathrm{ZnO}$ formed in a rotating magnetic field with various rotation speeds," Journal of the European Ceramic Society, vol. 29, no. 5, pp. 955-959, 2009.

[19] L. B. Duan, G. H. Rao, Y. C. Wang, J. Yu, and T. Wang, "Magnetization and Raman scattering studies of (Co,Mn) codoped ZnO nanoparticles," Journal of Applied Physics, vol. 104, no. 1, Article ID 013909, 5 pages, 2008.

[20] Y. Huang, M. Liu, Z. Li, Y. Zeng, and S. Liu, "Raman spectroscopy study of $\mathrm{ZnO}$-based ceramic films fabricated by novel sol-gel process," Materials Science and Engineering B, vol. 97, no. 2, pp. 111-116, 2003.

[21] N. H. Hong, J. Sakai, N. T. Huong, N. Poirot, and A. Ruyter, "Role of defects in tuning ferromagnetism in diluted magnetic oxide thin films," Physical Review B, vol. 72, no. 4, Article ID 045336, 5 pages, 2005.

[22] M. Venkatesan, C. B. Fitzgerald, J. G. Lunney, and J. M. D. Coey, "Anisotropic ferromagnetism in substituted zinc oxide," Physical Review Letters, vol. 93, no. 17, Article ID 177206, 4 pages, 2004.

[23] K. R. Kittilstved, D. A. Schwartz, A. C. Tuan, S. M. Heald, S. A. Chambers, and D. R. Gamelin, "Direct kinetic correlation of carriers and ferromagnetism in $\mathrm{Co}^{2+}: \mathrm{ZnO}$," Physical Review Letters, vol. 97, no. 3, Article ID 037203, 4 pages, 2006.

[24] Z. Jin, T. Fukumura, M. Kawasaki et al., "High throughput fabrication of transition-metal-doped epitaxial $\mathrm{ZnO}$ thin films: a series of oxide-diluted magnetic semiconductors and their properties," Applied Physics Letters, vol. 78, no. 24, pp. 3824-3826, 2001. 

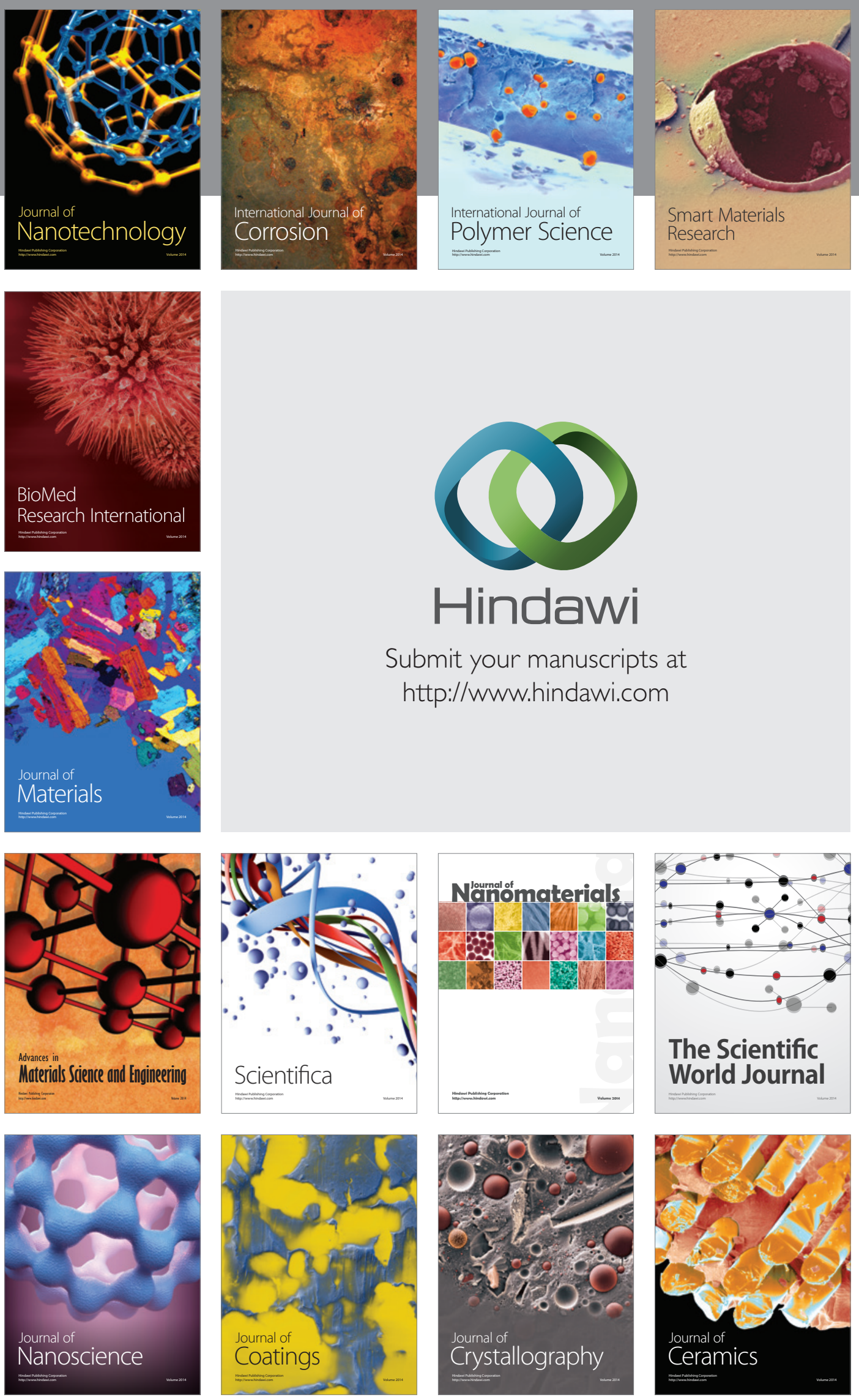

The Scientific World Journal

Submit your manuscripts at

http://www.hindawi.com

\section{World Journal}

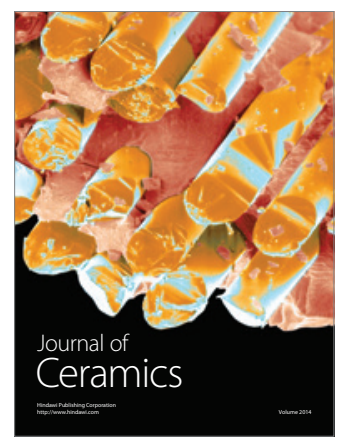

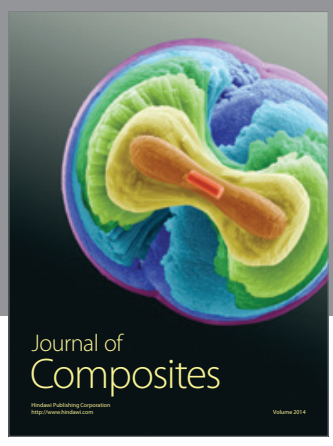
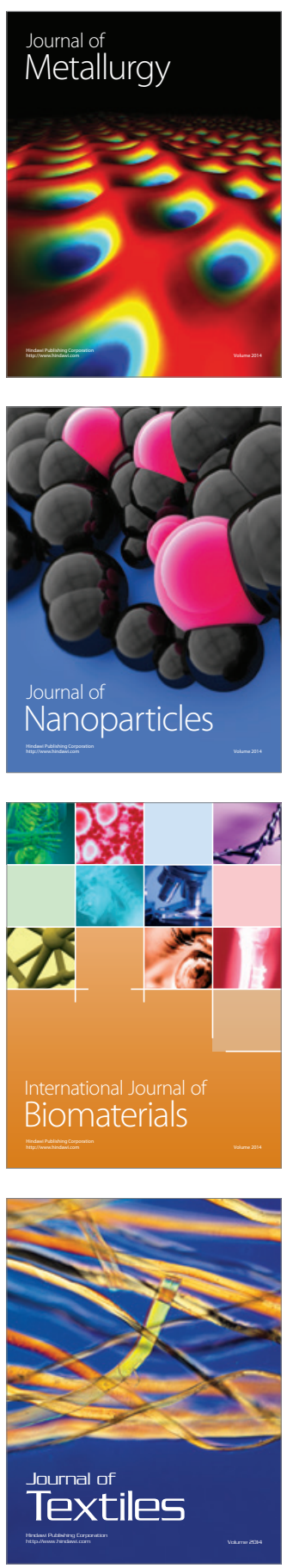